\title{
Pharmacists' Involvement in Diabetic Care
}

\author{
Muhammed Ansil ${ }^{1}$, Muhammad $\mathrm{Sadik}^{2}$, Kabeer $\mathrm{Pk}^{2}$, Ajay K raj², and Amal Manzoor ${ }^{2}$ \\ ${ }^{1} \mathrm{KMCT}$ Group of Educational Institutions \\ ${ }^{2} \mathrm{KMCT}$
}

July 20, 2020

\begin{abstract}
Objectives \&Introduction: Diabetes Mellitus (DM) is an emerging chronic disease with global significance . This article reviews 35 RCTs to identify the benefits of pharmacists' involvement in diabetic care in reducing the risk of diabetic complications and/or disease progression. Methods: 35 Randomized Controlled Trials (RCTs) were picked from 3210 publications listed on PubMed, using the keywords: diabetes, pharmacist, interventions and complications. Results of the studies were analysed and interpreted. Result: Interventions made by Pharmacists offer a potential benefit in achieving the goal of therapy in diabetes, perhaps by improving medication adherence and minimising diabetes-related complications significantly. Hence this review concludes that pharmacists can potentially play a pivotal role in the management of DM and maintain their QOL. In developing countries with poor record of pharmaceutical services, it is important to highlight the role of pharmacists in achieving rational and evidence-based pharmacotherapy. Conclusion: This study emphasizes Pharmacists' vital position in diabetic patient care. Pharmacists improve adherence to the drug, quality of life, cost-effectiveness of health care in patients with diabetes. These findings support the irreplaceable role of pharmacists in the diabetic care.
\end{abstract}

\section{Pharmacists' Involvement in Diabetic Care}

Muhammed Ansil T.P ${ }^{1}$, Kabeer PK ${ }^{1}$, Muhammad Sadiq ${ }^{1}$, Amal Manzoor ${ }^{1}$, Ajay K Raj ${ }^{1}$

Department of Pharmacy Practice, National college of pharmacy, Manassery, Kozhikode.

Corresponding Author:

Name: Muhammed Ansil TP

Email: muhammedansil007@gmail.com

Address: KMCT Group of Educational Institutions, Pharmacy Practice

Manassery

Kozhikode, Kerala, IN 673602

Phone: 9847648823

\section{ABSTRACT}

\section{Objectives \&Introduction:}

Diabetes Mellitus (DM) is an emerging chronic disease with global significance. This article reviews 35 RCTs to identify the benefits of pharmacists' involvement in diabetic care in reducing the risk of diabetic complications and/or disease progression.

\section{Methods:}


35 Randomized Controlled Trials (RCTs) were picked from 3210 publications listed on PubMed, using the keywords: diabetes, pharmacist, interventions and complications. Results of the studies were analysed and interpreted.

\section{Result:}

Interventions made by Pharmacists offer a potential benefit in achieving the goal of therapy in diabetes, perhaps by improving medication adherence and minimising diabetes-related complications significantly. Hence this review concludes that pharmacists can potentially play a pivotal role in the management of DM and maintain their QOL. In developing countries with poor record of pharmaceutical services, it is important to highlight the role of pharmacists in achieving rational and evidence-based pharmacotherapy.

\section{Conclusion:}

This study emphasizes Pharmacists' vital position in diabetic patient care. Pharmacists improve adherence to the drug, quality of life, cost-effectiveness of health care in patients with diabetes. These findings support the irreplaceable role of pharmacists in the diabetic care.

\section{What's Known:}

Pharmacists improve adherence to the drug, quality of life, cost-effectiveness of health care in patients with diabetes. These findings support the irreplaceable role of pharmacists in the diabetic care.

\section{Whats's New:}

This article emphasise the irreplaceable role of pharmacists in patient care and how their care helps those in need.

\section{INTRODUCTION}

Diabetes Mellitus (DM) is a chronic disorders characterized by increased blood glucose due to the impairment in insulin secretion, insulin activity or both. Diabetes is associated with long term damage, dysfunction, and impairment of various organs. ${ }^{1}$ Two major types of DM are Type 1 Diabetes Mellitus (T1DM) and Type 2 Diabetes Mellitus (T2DM). T1DM arises from $\beta$-cells destruction, resulting in total insulin deficiency, and requires external administration of insulin. T2DM is the result of progressive insulin loss and insulin resistance, which is nothing but ineffective action of insulin. DM is an emerging chronic disease that affect all ages, around the world. ${ }^{2}$ Global prevalence of diabetes has risen to 425 million in 2014, and is projected to rise to 629 million by $2045 .^{3}$

Pharmacist is not only a manufacturer or supplier of medicines, but also a care giver. FIP (International Pharmaceutical Federation) seven star concept of pharmacist assigns multiple roles to the pharmacists, including that of caregiver, communicator, decision maker, teacher, lifelong learner and manager. ${ }^{4}$ The patientcentred role of pharmacist is mounting in the context of many emerging new medicines and their negative consequences. ${ }^{4}$ The patient-centred role of pharmacist is mounting in the context of many emerging new medicines and their negative consequences. Clinical pharmacists are drug specialists; they should work together with other health professionals and plan a scheme to upgrade the well-being of the diabetic community worldwide. ${ }^{5}$ Pharmacists are more accessible to patients than other health care professionals in the community and are ideal health care partners for imparting knowledge to the patient and serving them through medication therapy management, health screening, emergency response and immunisation programs. These patient-centered activities of pharmacists are collectively known as pharmaceutical care (PC). A recent investigation has reported that the professional value of pharmacists is underutilized. Nonetheless, there were confirmatory reports from many nations that pharmacists have been contributing significantly to management of chronic diseases by monitoring, counselling and providing medication therapy. The profession of clinical pharmacy is new developing countries. Therefore, patient-centered services by pharmacists were initiated only very recently in countries like India. Indian pharmacists offer very little professional service to the patient, beyond the traditional activities of dispensing. Pharmacists receive training in therapeutics in 
addition to pharmacological characteristics of drugs such as indication, interactions, ADR, dosing, substitution etc. Pharmacists receive training in therapeutics in addition to pharmacological characteristics of drugs such as indication, interactions, ADR, dosing, substitution etc. Extensive training given to pharmacists is wasted because they play a very minor role in pharmacotherapeutic management. ${ }^{6-8}$

The pharmacist can help diabetic patients by different interventions such as monitoring of drug interactions in polypharmacy, assisting and advising patients regarding proper usage of pharmaceuticals and ADR reporting etc. Pharmacists' duties in diabetic care has expanded considerably in the task of accomplishing the ideal glucose level and other clinical outcomes. ${ }^{9}$ This review focuses on diabetic management in the collaborative care involving pharmacists and its potential impact on different outcomes.

\section{METHODOLGY}

We selectively chose papers from literature indexed in PubMed between 2005 and 2019, using the following keywords: diabetes, pharmacist, interventions and complications.

\section{RESULTS}

Of the 35 selected Randomized Control Trials, 3397 participants were randomly divided into two groups; one receiving pharmaceutical treatment and one receiving other normal treatment. Based on the following diabetic findings, the effect of pharmacists' strategies and patient-centered services was analyzed:-

- Clinical improvement.

- Quality of life (QOL).

- Health care cost effectiveness.

- Medication adherence / compliance.

\section{Clinical improvements and Management of complications in DM}

Monitoring and management for complications can significantly affect the quality of life (QOL) of diabetic patients. Early detection of complications can successfully reduce both mortality and seriousness of complications. Diabetic complications can be managed through recommendations for modifying lifestyle, alternative therapy, and pharmacological therapy.

Cardiovascular disease (CVD) is a leading source of mortality for diabetic patients. ${ }^{10,11}$ Therefore beyond the glycemic control, ideal diabetic management includes blood pressure and cholesterol control as well as monitoring of early neuropathic complications. Foot exams, comprehensive counselling and lifestyle modifications were also found to be critical. Extended pharmacy care improves hyperglycemic condition, in addition to decreasing cardiovascular risk in diabetes. ${ }^{12}$ Pharmacists' education program in diabetic patients significantly decreased cardiovascular risk by reducing total cholesterol and Low Density Lipoproteins (LDL) cholesterol. ${ }^{13}$

A Brazilian RCT (2011), in six primary care units, enrolling 200 patients, observed that prevalence of cardiac complications in elderly diabetic patients decreases with three years of pharmaceutical care. ${ }^{14}$ The pharmaceutical counselling programs resulted in a marked improvement in $\mathrm{HbA} 1 \mathrm{C}$ and $\mathrm{BP} .{ }^{15} \mathrm{~A}$ similar study (2011) from Rhode Island, USA revealed that pharmaceutical care was very highly effective for controlling hyperglycemia and hyperlipidemia in diabetic patients, more than those who received standard care by physicians only. A nine month study on 105 diabetic patients in Hong-Kong(2011) compared the impact of pharmacists' counselling with routine medical care. Individuals who received pharmaceutical care had lower CVD risk, LDL cholesterol and stroke. ${ }^{17}$

A study(2015) conducted in Germany with 65 T1DM adolescents was reported a marked improvement in $\mathrm{HbA1C}$ from pharmacist-led programs. ${ }^{18}$ Patients who received pharmacists' services increased their knowledge test score compared with baseline values, suggesting that pharmacists can potentially improve clinical condition. ${ }^{19-22}$

\section{Pharmacists care and QOL of diabetic patients}


Patients' adherence to medication and lifestyle modification are key factors in the management of diabetic complications that affect the patient's QOL. The complications of diabetes badly influence the QOL of a patient. Knowledge about disease condition and its management can improve the success of self care in DM and thereby improve QOL. Patient therapy on glycemic control can prevent or delay diabetic complications.23, 24

Randomized study on QOL in 240 T2DM patients (2009),showed that intervention by pharmacists adequately improved QOL when compared to usual care group. This was evaluated with 12 month follow-up by SF36 (ShortForm36) questionnaire. ${ }^{25}$ Different tools were used to evaluate QOL in diabetic patients (e.g. Diabetes Quality of life questionnaire.) Pharmacists based approach to the management of disease were effective and improved the patients QOL. ${ }^{11}$ A study conducted on 113 Indian diabetic patients confirmed the improvement in patient's well-being through pharmacist-initiated activities like counselling and education programs. The QOL was assessed by WHO-Bref QOL questionnaire. ${ }^{26}$ A 12 month RCT in Nigerian diabetic patients (2013) showed improvement in overall QOL when they received pharmaceutical care. ${ }^{27}$

\section{Health care cost effectiveness}

Cost is an important hurdle in the management of chronic diseases such as diabetes. Healthcare cost of diabetic patients includes the cost of continuing medications, complications' management, hospitalisation, drug-related problems, hospital visits and frequent follow ups, etc. The clinical improvement from an ideal diabetic care reduces direct medical costs. Selected studies use analytical techniques such as cost-benefit analysis, cost-effectiveness analysis and cost-consequences analysis for economic evaluation in health care. ${ }^{28-30}$

In 2014, 197 diabetic patients in Western Australia, were subjected to a study on cost-effectiveness of a pharmacist-led Diabetes Management Education Program (DMEP) in demonstrating clinical improvement. After 6-months follow-up, days with hyperglycemia decreased by 1.86 days per patient per month, resulting in a savings of US $\$ 39$ per day of hyperglycemia avoided. ${ }^{31} \mathrm{~A}$ trial with $123 \mathrm{~T} 2 \mathrm{DM}$ patients in Canada in 2014, pharmacist involvement in the management of diabetes provided a measurable reduction in the healthcare costs for post-intervention period. Patient-centered services provided by pharmacists have been found to lower the annual over-all health care cost of $\$ 190$ per patient when compared with usual care. ${ }^{8}$

In a US study(2017) with 250 diabetic patients, significant reduction was observed in health care costs of interventional group, after 13 -months follow-up. ${ }^{10}$ An average cost effectiveness of $\$ 91.01$ per patient who received collaborative care with pharmacist were found in a six-months study(2017) conducted in Singapore. ${ }^{3333}$ The Pharmacists' contribution to minimizing healthcare cost of diabetic patients is measured and the intervention group shows a decline in total direct medical costs. ${ }^{33}$

\section{Patient compliance to medication}

Medication adherence or compliance is the extent to which patients follow the prescription. Pharmacists can improve patient compliance to medication by various interventions. Different reasons may contribute to non adherence including polypharmacy, adverse reactions, frequency of medication and inconvenience of administration.

Adherence to or compliance with medication is the degree to which patients obey the prescription. Via various measures pharmacists can increase patient compliance with medication. Various factors can lead to non-compliance including polypharmacy, adverse reactions, prescription frequency and inconvenience of administration. Early onset of diabetic complications and poor QOL that result in non-adherence to the medication. The best tool for improving adherence is patient therapy on diabetes medication. Pharmacists can provide guidance on drug usage, storage, prevention, lifestyle modifications, diet and adverse effects to patients or their caregivers. Counselling will improve therapeutic outcomes and ensure the appropriate use of medication.34-37

A 12-month research (2018) in France enrolling 377 diabetic patients evaluated the efficacy of medication adherence therapy offered by pharmacist in patients with DM. Clinical pharmacist therapy of patients resulted in a significant improvement in prescription adherence rate, indicating greater compliance with 
medication38. In the Malaysian healthcare system; Diabetic Drug Therapy Adherence Clinic (DMTAC) offers services to pharmacists to strengthen adherence to the prescription. An RCT conducted in Malaysia in 2016 found that the community of diabetic patients led by pharmacists enhanced medication adherence relative to the control group.19 The study in Malaysia (2014) with 241 diabetic patients found that the model of pharmaceutical treatment would enhance adherence to medication.39 From an Iranian study in 172 T2DM patients (2009), In diabetic patients who obtained pharmaceutical treatment, an improvement in mean Morisky score and drug possession ratio was observed.40 Patient therapy performed by pharmacist has a major effect on the glycemic regulation of DM patients. This was confirmed by research performed in New York in 2015 and 2013 with 526 and 170 diabetic patients, respectively.

\section{DISCUSSION}

Pharmacist-led services greatly enhance diabetic patients' health status, quality of life, cost efficiency and adherence to medication. Internationally, 35 randomized clinical trials have been examined including 24 studies suggesting changes in QOL of pharmaceutical services. Other 11 research have showed that the intervention groups had substantial changes in assessed results relative to control or normal groups of treatment. The studies were performed at different healthcare facilities in various countries. Statistics endorse the role of the pharmacist as a health care professional in diabetes management.

The presence of pharmacists indicated a significant increase in HbA1C, lipid profiles, blood pressure, intervention group BMI. There have been substantial variations between monitoring outcomes and the intervention classes. In patients exposed to prescription facilities, the adherence rate to medications also increased. Pharmaceutical treatment was cost-effective with significant decline in the expense of diabetic patients in health care. Each result was evaluated by periodic follow-up during the study period and compared with the initial baseline. Specific approaches by pharmacists focused on different pharmacist duties and responsibilities in diabetes management settings. More studies are required to identify the exact reason for the growing incidence of diabetes, and to prepare for successful complication prevention.

\section{CONCLUSION}

Pharmacists' strategies provide a possible advantage in achieving the goal of diabetes care, perhaps by dramatically increasing adherence to the drug and reducing problems related to diabetes. This review concludes therefore that pharmacists can potentially play a pivotal role in managing DM and maintaining their QOL. It is necessary to emphasize the position of pharmacists in achieving fair and evidence-based pharmacotherapy in developed countries with poor record of pharmaceutical services.

\section{CONFLICTS OF INTEREST}

There are no conflicts of interest regarding this work of study.

\section{LIMITATIONS}

This review included studies were evaluated different types of interventions and conducted in various healthcare settings. Therefore, limitations of the study are the following:

- Increased level of heterogeneity of the results.

- Different tools are used for assessment of some outcomes.

\section{ACKNOWLEDGEMENT:}

We are thankful to all the staffs in the hospital who supported our work.

\section{REFERENCE}

1. World Health Organization. Definition, diagnosis and classification of diabetes mellitus and its complications: report of a WHO consultation. Part 1, Diagnosis and classification of diabetes mellitus. Geneva: World Health Organization; 1999. http://apps.who.int/iris/handle/10665/66040. Accessed April 28, 2019. 
2. Roglic G. WHO Global report on diabetes: A summary. Int J Non-Commun Dis. 2016;1(1):3.

3. International Diabetes Federation. IDF Diabetes Atlas . $8^{\text {th }}$ ed. Brussels, Belgium: International Diabetes Federation; 2018. http://www.idf.org/diabetesatlas. Accessed April 30, 2019.

4. Wiedenmayer K, Summers RS, Mackie CA, et al . Developing pharmacy practice: focus on patient care: Handbook. Geneva: World Health Organisation; 2006. http://www.who.int/medicines/publications/WHO_PSM_PAR_2006.5.pdf. Accessed May 2, 2019.

5. Mossialos E, Courtin E, Naci H, et al. From "retailers" to health care providers: transforming the role of community pharmacists in chronic disease management. Health policy . 2015;119(5):628-639. doi: 10.1016/j.healthpol.2015.02.007

6. Khanal S, Nissen L, Veerman L, Hollingworth S. Pharmacy workforce to prevent and manage non-communicable diseases in developing nations: The case of Nepal. Res Social Adm Pharm . 2016;12(4):655-659.

7. Deshpande PR, Vantipalli R, Lakshmi CC, et al . Clinical pharmacists: The major support to Indian healthcare system in near future. J Pharm Bioallied Sci . 2015;7(3):161-174.

8. Simpson SH, Lier DA, Majumdar SR, et al . Cost-effectiveness analysis of adding pharmacists to primary care teams to reduce cardiovascular risk in patients with Type 2 diabetes: results from a randomized controlled trial.Diabetic Med. 2015;32(7):899-906. doi: 10.1111/dme.12692.

9. Upadhyay DK, Ibrahim MI, Mishra P, Alurkar VM. A non-clinical randomised controlled trial to assess the impact of pharmaceutical care intervention on satisfaction level of newly diagnosed diabetes mellitus patients in a tertiary care teaching hospital in Nepal.BMC health serv. Res. 2015;15(1):57-67.

10. Wu WC, Taveira TH, Jeffery S, Jiang L, Tokuda L, Musial J, Cohen LB, Uhrle F. Costs and effectiveness of pharmacist-led group medical visits for type-2 diabetes: A multi-center randomized controlled trial. PLoS One . 2018;13(4):e0195898.

11. Wishah RA, Al-Khawaldeh OA, Albsoul AM. Impact of pharmaceutical care interventions on glycemic control and other health-related clinical outcomes in patients with type 2 diabetes: randomized controlled trial. Diabetes MetabSyndr . 2015;9(4):271-276.

12. Aguiar PM, da Silva CH, Chiann C, et al . Pharmacist-physician collaborative care model for patients with uncontrolled type 2 diabetes in Brazil: results from a randomized controlled trial.J Eval Clin Pract. 2018;24(1):22-30.

13. Kiel PJ, McCord AD. Pharmacist impact on clinical outcomes in a diabetes disease management program via collaborative practice.Ann Pharmacother . 2005;39(11):1828-1832.

14. Obreli-Neto PR, Guidoni CM, de Oliveira Baldoni A, et al . Effect of a 36-month pharmaceutical care program on pharmacotherapy adherence in elderly diabetic and hypertensive patients. Int Jof clin pharm . 2011;33(4):642-649.

15. Al Hamarneh YN, Hemmelgarn BR, Hassan I, Jones CA, Tsuyuki RT. The effectiveness of pharmacist interventions on cardiovascular risk in adult patients with type 2 diabetes: the multicentre randomized controlled RxEACH trial. Can J Diabetes . 2017;41(6):580-586.

16. Cohen LB, Taveira TH, Khatana SA, et al . Pharmacist-led shared medical appointments for multiple cardiovascular risk reduction in patients with type 2 diabetes. Diabetes Educ . 2011;37(6):801-812.

17. Chan CW, Siu SC, Wong CK, Lee VW. A pharmacist care program: positive impact on cardiac risk in patients with type 2 diabetes. J Cardiovasc Pharmacol Ther . 2012;17(1):57-64.

18. Obarcanin E, Krüger M, Müller $\mathrm{P}$, et al . Pharmaceutical care of adolescents with diabetes mellitus type 1: the DIADEMA study, a randomized controlled trial. Int J Clin Pharm.2015;37(5):790-798.

19. Lim PC, Lim K, Embee ZC, et al . Study investigating the impact of pharmacist involvement on the outcomes of diabetes medication therapy adherence program Malaysia. Pak J Pharm Sci . 2016;29(2):595601.

20. Anderegg MD, Gums TH, Uribe L, et al . Pharmacist intervention for blood pressure control in patients with diabetes and/or chronic kidney disease. Pharmacotherapy . 2018;38(3):309-318.

21. Bukhsh A, Nawaz MS, Ahmed HS, Khan TM. A randomized controlled study to evaluate the effect of pharmacist-led educational intervention on glycemic control, self-care activities and disease knowledge among type 2 diabetes patients. Med . 2018;97(12):e9847. 
22. Jarab AS, Alqudah SG, Mukattash TL, Shattat G, Al-Qirim T. Randomized controlled trial of clinical pharmacy management of patients with type 2 diabetes in an outpatient diabetes clinic in Jordan. $J$ Manag Care Pharm . 2012;18(7):516-526.

23. Venkatesan R, Devi AS, Parasuraman S, Sriram S. Role of community pharmacists in improving knowledge and glycemic control of type 2 diabetes. Perspect Clin Res . 2012;3(1):26-31.

24. Taveira TH, Dooley AG, Cohen LB, Khatana SA, Wu WC. Pharmacist-led group medical appointments for the management of type 2 diabetes with comorbid depression in older adults. Ann Pharmacother . 2011;45(11):1346-1355.

25. Al Mazroui NR, Kamal MM, Ghabash NM, et al . Influence of pharmaceutical care on health outcomes in patients with type 2 diabetes mellitus. Br J Clin Pharmacol . 2009;67(5):547-557.

26. Ramanath KV, Santhosh YL. Impact of clinical pharmacist provided patient education on QOL outcome in type II diabetes mellitus in rural population. Asian J Pharm Clin Res . 2011;4(4):15-20.

27. Adibe MO, Ukwe CV, Aguwa CN. The impact of pharmaceutical care intervention on the quality of life of Nigerian patients receiving treatment for type 2 diabetes. Value Health Reg Issues . 2013;2(2):240247.

28. Chen JH, Ou HT, Lin TC, Lai EC, Kao YH. Pharmaceutical care of elderly patients with poorly controlled type 2 diabetes mellitus: a randomized controlled trial. Int J Clin Pharm. 2016;38(1):88-95.

29. Adibe MO, Aguwa CN, Ukwe CV. Cost-utility analysis of pharmaceutical care intervention versus usual care in management of Nigerian patients with type 2 diabetes. Value Health Reg Issues . 2013;2(2):189198.

30. Mehuys E, Van Bortel L, De Bolle L, et al . Effectiveness of a community pharmacist intervention in diabetes care: a randomized controlled trial. J Clin Pharm Ther . 2011;36(5):602-613.

31. Hendrie D, Miller TR, Woodman RJ, Hoti K, Hughes J. Cost-effectiveness of reducing glycaemic episodes through community pharmacy management of patients with type 2 diabetes mellitus. J Prim Prev . 2014;65(6):439-449.

32. Siaw MY, Ko Y, Malone DC, et al . Impact of pharmacist-involved collaborative care on the clinical, humanistic and cost outcomes of high-risk patients with type 2 diabetes (IMPACT): a randomized controlled trial. J Clin Pharm and Ther. 2017;42(4):475-482.

33. Kjeldsen LJ, Bjerrum L, Dam P, et al. Safe and effective use of medicines for patients with type 2 diabetes: a randomized controlled trial of two interventions delivered by local pharmacies. Res Social Adm Pharm . 2015;11(1):47-62.

34. Korcegez EI, Sancar M, Demirkan K. Effect of a pharmacist-led program on improving outcomes in patients with type 2 diabetes mellitus from Northern Cyprus: a randomized controlled trial. J Manag care Spec pharm . 2017;23(5):573-582.

35. Plaster CP, Melo DT, Boldt V, et al . Reduction of cardiovascular risk in patients with metabolic syndrome in a community health center after a pharmaceutical care program of pharmacotherapy follow-up. Braz J Pharm Sci . 2012;48(3):435-446.

36. Ali M, Schifano F, Robinson P, et al . Impact of community pharmacy diabetes monitoring and education programme on diabetes management: a randomized controlled study.Diabet Med . 2012;29(9):e326-333.

37. Adepu SP, Ramanath KV, Bhat S, Sam S, Omanakuttan C. Assessment of Pharmacist-Mediated Education on Medication Adherence Behaviour in Type 2 Diabetes Mellitus Patients. Value Health . 2016;19(3):A18.

38. Michiels Y, Bugnon O, Chicoye A, et al . Impact of a Community Pharmacist-Delivered Information Program on the Follow-up of Type-2 Diabetic Patients: A Cluster Randomized Controlled Study. Adv Ther . 2019;36(6):1291-1303.

39. Chung WW, Chua SS, Lai PS, Chan SP. Effects of a pharmaceutical care model on medication adherence and glycemic control of people with type 2 diabetes. Patient Prefer Adher . 2014;8:1185-1194.doi: 10.2147/PPA.S66619.

40. Farsaei S, Sabzghabaee AM, Zargarzadeh AH, Amini M. Effect of pharmacist-led patient education on glycemic control of type 2 diabetics: a randomized controlled trial. J Res Med Sci . 2011;16(1):43-49. 
41. Cohen HW, Shmukler C, Ullman R, Rivera CM, Walker EA. Measurements of medication adherence in diabetic patients with poorly controlled HbA1c. Diabetic Medicine. 2010;27(2):210-216.

42. Gonzalez JS, Schneider HE, Wexler DJ, et al. Validity of medication adherence self-reports in adults with type 2 diabetes. Diabetes Care . 2013;36(4):831-837. 